\title{
LA ENSEÑANZA DE LA ECOLOGÍA. UN OBJETIVO DE LA EDUCACIÓN AMBIENTAL
}

\author{
FERNÁNDEZ MANZANAL, R. Y CASAL JIMÉNEZ, M. \\ IB Hermanos D'Elhuyar (Logroño). \\ Departamento de Ecología de la Universidad de Santiago de Compostela (La Coruña).
}

\begin{abstract}
SUMMARY
In this article we study the repercussion that studies on ecology may have on environmental education. To do so, we review the student's alternative ideas about concepts and principles of ecology selected for the study of the subject. We stress the idea that outings to the countryside to study a specific ecosystem helps to clarify the interactions between the living things and the environment. Finally, we give the data of a research performed with high school students, supporting the idea that those students who carry out work in the countryside are able to define a larger number of components and relationships. Such training favours the transfer of ecology knowledge, both in the evaluation of an environmental problem and in proposing solutions.
\end{abstract}

\section{INTRODUCCIÓN}

La educación ambiental se está configurando en nuestra época como una de las necesidades más importantes de la formación de los ciudadanos. Como actividad pedagógica es bastante reciente, pero puede decirse que en un sentido más amplio surgió cuando el hombre comprendió su relación con la biosfera y empezó a cuestionarse su papel en la conservación y degradación del entorno (Hungerford y Peyton, 1992).

La amplitud de la degradación del medio (aguas de ríos y mares saturadas de contaminación, bosques y grandes reservas vegetales devastados, agotamiento de combustibles fósiles, liberación incontrolada de contaminantes atmosféricos, crecimiento desmesurado de las áreas utbanas y acumulación creciente de desechos, etc.) está adquiriendo una complejidad tal que ya desde finales de los sesenta empezaron a hacerse las primeras recomendaciones sobre la conveniencia de familiarizar a los ciudadanos con los problemas ambientales. Desde entonces, en diversas conferencias y congresos internacionales promovidos por la UNESCO (Programa MAB, Conferencia de Estocolmo, Seminario Internacional de Educación Ambiental de Belgrado, Conferencia de Tbilisi, Conferencia de Río, etc.) se ha becho hincapié en la importancia de formar profesionales cuyas actividades y decisiones influyan con especial significación en la calidad del medio ambiente. Entre las formas de actuación propuestas están lógicamente, y con especial relevancia, aquéllas que faciliten la intervención en el aula y que muestren a los profesores cuáles son los caminos más adecuados para hacer de los alumnos ciudadanos que se sientan implicados en el cuidado del medio. Así, en el informe final de la Conferencia de Tbilisi (Unesco, 1977) se dice: La educación ambiental no debe ser una asignatura más a añadir a los programas escolares existentes, sino que debe incorporarse a los programas destinados a los alumnos, cualquiera que sea su edad [...]. Esta labor precisa la aplicación de nuevos conceptos, de nuevos métodos y nuevas técnicas en el marco del esfuerzo global que destaca el papel social de las instituciones educativas y la creación de nuevas relaciones entre los participantes en el proceso educativo.

La educación ambiental, según Terradas (1979), es una forma de educación especialmente centrada en el entorno. Reconociendo que el entorno puede ser prácticamente todo y que esta palabra, por su ambigüedad, introduce múltiples dificultades operativas (Peters, 1991), hemos considerado conveniente introducir en esta investigación algunas restricciones al significado del término y 
aplicar el valor del mismo a cuestiones del entorno que pueden ser tratadas desde el marco conceptual que proporciona la ecología.

Indudablemente, la complejidad de los problemas ambientales no permite abordarlos con la estrechez de miras de las teorías que aporta una sóla disciplina científica, aunque ésta sea la ecología. Es necesario, en palabras de Giordan y otros (1993), ampliar nuestra preocupación hacia aspectos que engloben el medio urbano $e$ industrial para captar las relaciones de interdependencia. Gran parte de los esfuerzos se centran hoy en descubrir la trama de conexiones que los diversos conocimientos pueđen establecer en la comprensión global del funcionamiento de la Tierra y en la pervivencia del sistema. No obstante, es necesario también clarificar en qué medida cada disciplina, con sus centros de interés propios y sus particulares métodos de trabajo, puede contribuir a proporcionar la visión integradora que los fenómenos ambientales reclaman. Entonces, ¿qué aporta la ecología a la hora de facilitar la comprensión de las interacciones entre los componentes deI medio $y$, en particular, la influencia de la actuación humana? ¿En qué medida los conocimientos de ecología pueden contribuir a favorecer el cambio hacia posiciones más comprometidas con la defensa del entorno y de los seres vivos? ¿Cómo acercar los conocimientos de ecología a un gran sector de la población como el que forman los estudiantes de secundaria?

Para analizar estas cuestiones consideramos necesario delimitar los siguientes aspectos:

- importancia de la ecología en la educación ambiental;

- dificultades del aprendizaje de conceptos y principios de ecología entre estudiantes de secundaria;

- estrategias que pueden favorecer la superación de alguna de las dificultades analizadas;

- cómo influye el trabajo de campo en la concepción global del ecosistema y en la educación ambiental.

Pasaremos, a continuación, a comentar algunos detalles de estos apartados.

\section{LA ECOLOGÍA EN LA EDUCACIÓN AMBIENTAL}

Hoy en día se asume que los estudios de ecología constituyen un componente básico de la educación ambiental (Booth, 1979). Las orientaciones de la LOGSE para los nuevos diseños curriculares presentan algunas recomendaciones para la educación ambiental, como tema transversal del currículo en este caso, en que los conceptos y principios de ecología parecen constituir uno de los pilares esenciales para la promoción de la educación ambiental entre estudiantes de Secundaria (Jiménez y Laliena, 1992). Así mismo Hungerford y Peyton (1992), al definit las metas de los programas de educación ambiental, consideran que en la formulación de objetivos se debe seguir un orden tal que permita ampliar progresivamente los conocimientos y competencias para actuar de forma comprometida con la defensa del medio. En este orden de objetivos figura en el nivel I el estudio de los elementos de ecología que, según los autores, permitan tomar, llegado el momento, decisiones ecológicamente racionales de cara a los problemas ambientales. En el dominio científico, señala Gagliardi (Gagliardi, Martinand y Souchon, 1991), los conceptos de ecología representan conceptos esenciales para Ia educación ambiental, tanto por los contenidos como por la forma de abordarlos.

El valor de la ecología se apoya, a nuestro entender, en que aporta los elementos básicos para la comprensión de las relaciones de la especie humana con su entorno. Como señala Margalef (1974), los problemas de conservación y explotación de la naturaleza son básicamente ecológicos y deben enfocarse más desde un punto de vista educativo que formulando leyes y reglamentos. Conscientes de ello son numerosas las reuniones y organismos internacionales que han recomendado presentar de forma apropiada los principios fundamentales de ecología en los distintos niveles de la educación.

No se trata, en esencia, de añadir nuevos temas al currículo de las ciencias naturales sino de dar una visión más acorde con las demandas de la sociedad actuai (Gasyford, 1989). Cabe, por tanto, hacer girar los contenidos hacia la perspectiva de la interrelación de todos los componentes mediante el reforzamiento de los estudios que marquen dicha orientación. Los conocimientos de ecología pueden contribuir a promover actitudes favorables hacia el medio en la medida en que incrementen la capacidad de los estudiantes para comprender la relación de la especie humana con la biosfera.

Pero la educación ambiental tiene, como venimos señalando, sus propios objetivos. La adquisición de conocimientos de ecología no sería garantía suficiente para un cambio duradero de actitudes y conducta ambientalista si los mismos no fueran acompañados de experiencias que involucren, además de la inteligencia, otros aspectos de los alumnos, como sus emociones, vivencias del medio en un ambiente de aprendizaje, etc. De acuerdo con ello, queremos mostrar que es posible generar actitudes que orienten las decisiones a favor del medio ambiente si a la hora de estudiar ecología se proponen contenidos sobre el medio y estrategias de aprendizaje $a$ través del medio. Como señala Colom Cañellas (1993), la incidencia en la educación ambiental se producirá as í en la esfera de lo axiológico, en que el mundo de los valores constituye su verdadera especificidad. El proolema se plantea a la hora de seleccionar el conjunto de principios de ecología o el método de trabajo para favorecer la comprensión de la interdependencia de los seres vivos y el medio. Algunas de las dimensiones de la definición del problema tienen que ver con el modelo de estudio de ecología que se adopte, pero otras están directamente relacionadas con los obstáculos que tienen Ios estudiantes para su aprendizaje. Abordaremos, a continuación, este último aspecto. 


\section{DIFICULTADES QUE PRESENTA LA ENSENANZA/APRENDIZAJE DE LA ECO- LOGIA EN EL BACHILLERATO}

Al igual que en otras ciencias, es esencial precisar el tipo de componentes y relaciones que los estudiantes pueden establecer para la comprensión de los principios básicos de ecología con el fin de que, como expresa Astolfi (1987), se puedan tomar decisiones sobre el entramado de dificultades con las que se quiere que trabajen los alumnos para alcanzar un progreso identificable, incluso si éste es parcial y debe ser seguido de progresos suplementarios ulteriores.

La importancia atribuida a las ideas previas de los estudiantes ha favorecido el desarrollo de una de las líneas de investigación más fecundas de los últimos años, como bien conocen los lectores de esta revista. En diferentes publicaciones se han mostrado no sólo las ideas alternativas de los estudiantes en diversos campos, sino que se han analizado también las causas de las mismas, así como su falta de correspondencia con las concepciones científicas. No obstante, los trabajos de didáctica de la ecología referidos a la percepción global de las interacciones han sido menos numerosos. Por ello, nos detendremos en el próximo apartado en el análisis de dichas concepciones.

\section{Ideas alternativas de los estudiantes sobre conceptos y principios de ecología}

Una parte del trabajo objeto de este artículo se basó en reconocer el punto de partida de las alumnas y alumnos con los que se realizó el estudio de ecología, estudiantes de $1^{2}$ de BUP. Esta exploración se hizo extensiva a estudiantes de otros niveles educativos, lo cual permitió constatar que gran parte de las ideas alternativas encontradas entre alumnos de bachillerato, en lo que a conceptos de ecología se refiere, persisten entre alumnos con estudios más profundos de biología.

La muestra la constituyeron 7 grupos de estudiantes: 3 de

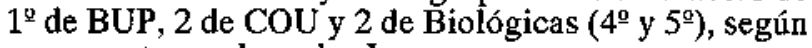
se presenta en el cuadro 1 .

\section{Cuadro I}

Distribución de la población investigada por niveles de escołaridad.

\begin{tabular}{|lccc|}
\hline Nivel educativo & Año & N $^{2}$ grupos & Total alurnnos \\
\hline $1^{\circledR}$ BUP & $1989-90$ & 1 & 34 \\
$1^{\circledR}$ BUP & $1990-91$ & 2 & 67 \\
COU & $1989-90$ & 2 & 64 \\
$4^{1} / 5^{\circledR}$ Biológicas & $1990-91$ & 2 & 75 \\
\hline
\end{tabular}

Los alumnos de $1^{2}$ de BUP no habían estudiado previamente ni los componentes ni la estructura de los ecosistemas. Todos los estudiantes de COU habían cursado la asignatura de Ciencias Naturales el primer año de sus estudios de bachillerato que, como se sabe, figura en el plan de 1974 como asignatura obligatoria e incluye los temas de ecología en la programación. Mediante la aplicación de las pruebas a estudiantes de COU y universitarios perseguíamos una doble finalidad: por un lado, establecer el nivel de confianza de los tests aplicados; por otro, reconocer categorías en las respuestas para cada una de las cuestiones en función de las concepciones alternativas implícitas. Así pues, nos interesa señalar que, aunque con esta exploración no se trataba de analizar las ideas alternativas de los estudiantes de estos niveles, una consecuencia de la búsqueda de categorías de respuesta fue el descubrimiento de dichas concepciones.

\section{Características del cuestionario}

Para comprobar la situación inicial de los estudiantes de $1^{2}$ de BUP, recurrimos a un estudio descriptivo (análisis de contenido) de las respuestas emitidas a un cuestionario preparado al efecto en el que se emplearon instrumentos de diverso tipo. Los que vamos a analizar para estos comentarios reponden, básicamente, a dos modelos: cuestiones de elección múltiple y problemas de respuesta abierta.

El contenido de las cuestiones se refiere a tres tipos de conceptos: los que atanen a las nociones consideradas preliminares para abordar otras más complejas de ecología, los que se refieren a las funciones de los componentes de los ecosistemas y los que tienen que ver con las relaciones tróficas. (Consideramos conceptos preliminares aquéllos cuyo desconocimiento impediría o dificultaría enormemente el aprendizaje de aspectos organizativos del ecosistema. Por ejemplo: el significado de animal o vegetal; es decir, saber aplicar a los mismos las características científicas que permitan comprender su distinta organización y función celular).

\section{Nociones preliminares}

Para reconocer las características que los estudiantes eligen en la definición de animales o vegetales nos basamos en una cuestión planteada por Bell (1981), de cuya réplica y resultados han aparecido referencias en los trabajos de Bell y Barker (1982), Trowbridge y Mintzes (1988) o, más recientemente, Velasco (1991). Los ejemplos que los estudiantes debían clasificar como animales o vegetales eran los siguientes: una figura humana, una planta con flores, una rana, una planta sin flores, un pájaro, un árbol, una estrella de mar y una lombriz de tierra.

La cuestión planteada por nosotros presenta ligeras modificaciones respecto a la de Bell, pero dado que las variaciones introducidas no cambian sustancialmente la idea original y que los resultados de las concepciones de los estudiantes son semejantes a los obtenidos por los autores citados, señalaremos, a modo de resumen, cuatro de las conclusiones de esta exploración. 
- La tendencia más acusada de clasificación entre los estudiantes de todos los niveles es la de aplicar a los animales o vegetales características bien visibles en los ejemplos presentados, o derivadas deI reconocimiento de su hábitat. Por ejemplo: "tiene alas", "vive en la tierra".

- La mayor parte de los estudiantes de bachillerato, y aun de etapas superiores, tiende a diferenciar a los animales a partir de los rasgos más visibles de los vertebrados, en particular de las aves y de los mamíferos. Por ejemplo, "los animales tienen patas", "respiran por pulmones", "emiten sonidos", etc.

- Las características más sobresalientes de la clasificación vegetal tienen que ver, en consecuencia, con la negación de las propiedades atribuidas a los animales.

- De particular interés, en lo que a la caracterización de los vegetales se refiere, es la definición de los mismos a través del enunciado "se alimenta de tierra".

Como destacan los autores citados, es importante percatarse de esta situación, pues en muchas ocasiones profesores y alumnos empleamos las mismas palabras con distinto significado. Para Bell y Barker (1982), es esencial que los estudiantes entiendan el significado de los términos animal y vegetal si queremos que comprendan el papel que desempeñan en los ecosistemas.

\section{Función de los componentes de los ecosistemas}

Para precisar el conocimiento de la función de los componentes de Ios ecosistemas planteamos tres cuestiones. En una de ellas, que se presenta en el anexo I, solicitábamos la identificación de los constituyentes de una cadena alimentaria con su nivel trófico.

A la hora de valorar esta cuestión, y la siguiente que analizaremos, establecimos cinco categorías de respuestas aplicando a las mismas una escala ordinal de medida, de manera que la categoría primera (categoría A) fue para los miembros de la clase preferida, entendiendo que aludimos a una ejecución excelente como de primera clase, $y$ a las ejecuciones inferiores como de segunda, tercera... clases.

En el caso que ahora nos ocupa, la variedad de combinaciones de respuestas dificulta, en cierto modo, la organización de categorías. Por ello, se ha asignado la misma valoración a respuestas distintas en aquellos casos en que las situaciones presentadas muestran equivalencia a la hora de traducir dichos comentarios a determinadas concepciones. Las categorías se especificaron como sigue:

A. Asigna un ejemplo correcto a cada nivel trófico. No adjudica ningún ejemplo a descomponedor.

B. Asigna un ejemplo correcto a cada nivel trófico y adjudica un ejemplo a descomponedor.

C. Sólo se señalan dos niveles tróficos.
D. Muestra equivalencia entre consumidor y uno sólo de los niveles (herbívoro o carnívoro). Presenta como único consumidor a los humanos.

E. Muestra equivalencia entre productor y consumidoro entre productor y descomponedor. O no contesta.

En el gráfico I se presentan Ios resultados de las categorías establecidas en los tres niveles educativos.

Gráfico I

Interpretación de los niveles tróficos.

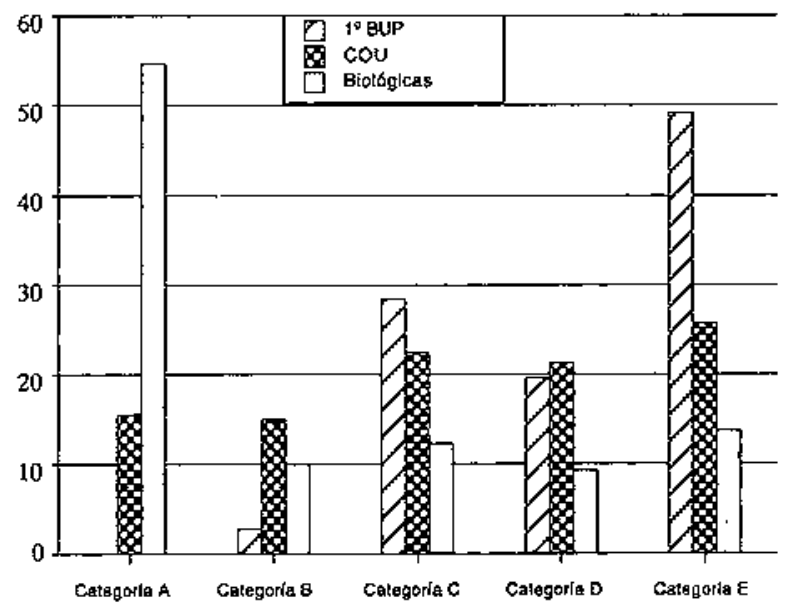

Las conclusiones más relevantes de esta exploración se pueden sintetizar como sigue:

- Ningún estudiante de la muestra de bachillerato conoce el significado de todos los términos solicitados. Así se puede entender si acordamos con Klausmeier (1976) que se muestra comprensión de un concepto cuando se puede actuar de forma clara y consistente respecto a la distinción entre ejemplos y contraejemplos. (Conviene indicar que se había ayudado a identificar, a todos estos estudiantes, la procesionaria y el cuco).

- La proporción de estudiantes englobados en la primera categoría aumenta a medida que aumenta el nivel educativo. No obstante, el hecho de que todas las categorías estén representadas cualquiera que sea la edad de los alumnos puede indicar que, a pesar de los estudios realizados, no se han clarificado algunos conceptos de la teoría ecológica.

- Los niveles más ampliamente reconocidos son los de herbivoros y carnívoros. El nivel productor sóto se presenta en los casos en los que hay una completa iđentificación de niveles, lo cual indica la poca importancia que se asigna a los vegetales en el mantenimiento de la vida. Esta idea viene a confirmar los resultados de 
otros estudios (Bell y Brook, 1984; Wandersee, 1985; Barker, 1985; Barker y Carr, 1989) que señalanl la falta de identificación de los vegetales con su función como organismos autótrofos, productores de la mayor parte de la materia orgánica de los ecosistemas.

- El importante papel asignado a la especie humana entre los consumidores parece más el resultado de con. sideratlo como un gran consumidor de productos técnicos, o en general de bienes de uso, que el derivado del significado ecológico.

- El hecho de que los humanos de las viñetas no estén incluidos en la cadena trófica representada $y$, a pesar de ello, sea un ejemplo muy citado en cualquiera de los niveles, nos lleva a concluir que los estudiantes que así se expresan no están analizando los niveles tróficos.

- También el concepto de descomponedor se muestra muy alejado de su acepción ecológica. Los ejemplos expuestos por los estudiantes inducen a pensar que los descomponedores son seres o sustancias dañinas que perjudican al conjunto de la cadena. Se incluye, entre los descomponedores: la procesionaria, en primer lugar; en menor proporción, la especie humana; $y$, por último, los venenos y el oxígeno.

Otras cuestiones analizadas, al igual que ésta, han ofre. cido resultados semejantes. Entre ellos destaca la escasa importancia asignada a los organismos autótrofos y la falta de correspondencia de los organismos descomponedores con su función en los ecosistemas.

\section{Relaciones tróficas}

Mostramos, a continuación, un tercer grupo de concep" ciones que se refieren a las relaciones alimentarias y, en general, a las relaciones entre los componentes bióticos. Los trabajos de Adeniyi (1985), Griffiths y Grant (1985), Peterfalvi, Rumelhard y Verin (1987), Humphreys (1987) y Akinsola (1990), entre otros, analizan la forma en que los estudiantes de diversos paises y edades perciben estas relaciones. La exploración realizada por nosotros se ha basado en el análisis de dos cuestiones de respuesta abierta, una de las cuales, que se recoge en el anexo II, pasaremos a comentar.

En el ejemplo propuesto se presentan cuatro niveles tróficos con figuras enlazadas mediante flechas que expresan la transferencia de energía alimentaria. Como se sabe, las cadenas de alimentos no son series aisladas. La complejidad de las uniones entre unas cadenas y otras en una comunidad se simboliza mediante complejos entramados de Ias especies componentes. Pero, dejando a un lado tales conexiones, pretendiamos averiguatr en qué medida los estudiantes comprenden las relaciones tróficas en los ecosistemas y cuáles son sus concepciones sobre la transferencia de materia y sobre los cambios bruscos de la disponibilidad de alimento.

Las categorías de respuestas, como en el caso anterior, se valoraron en función de una medida ordinal. Para esta- blecer dichas categorías se hizo previamente una detallada revisión de las respuestas de todos los estudiantes con el fin de que todas las explicaciones estuvieran representadas. La correspondencia con las explicaciones quedó como sigue:

A. Las poblaciones $\mathrm{C}$ y $\mathrm{D}$ decrecerán considerablemente hasta casi desaparecer. Aparece, sin embargo, referencia explícita o implícita a otras fuentes de alimentación.

B. Las poblaciones $\mathrm{C}$ y $\mathrm{D}$ se extinguirán o decrecerán. En este caso, no se hace referencia a otras fuentes de alimentación.

\section{Las poblaciones C y D se mantienen. Se adaptan.}

D. La población $D$ disminuirá porque se alimenta de $B$. No se comentan los efectos en la población C.

\section{E. Respuestas confusas, o bien no contesta.}

El resultado de la valoración realizada con los estudiantes de la muestra se presenta en el gráfico II.

\section{Gráfico II}

Porcentaje de respuestas de cada categoría correspondientes a la cuestión đe relaciones alimentarias.

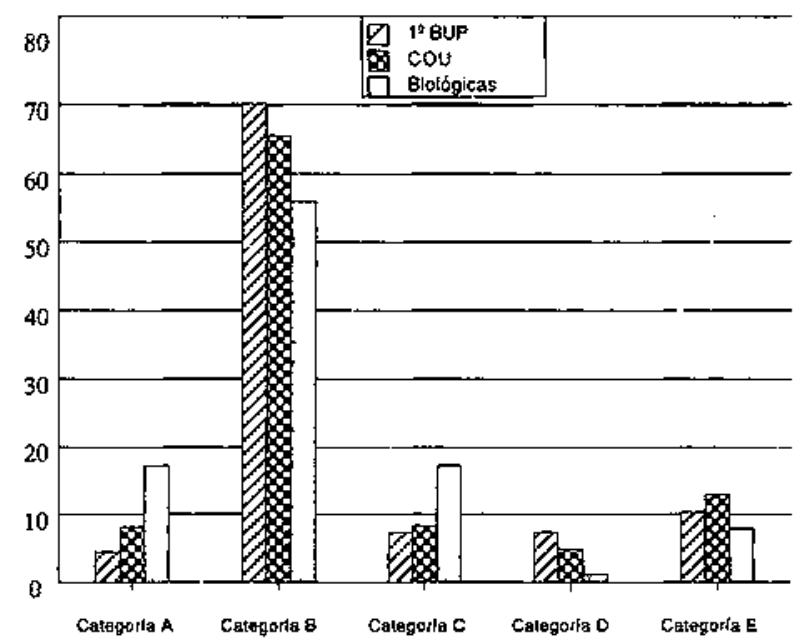

Las conclusiones más importantes obtenidas de esta exploración pueden resumirse como sigue:

- La mayoría de los estudiantes de todos los niveles educativos, incluidos Ios de bachillerato, se sitúan en la categoría B. Parecería, en consecuencia, que las relaciones derivadas del sistema depredador-presa son bien conocidas. Los estudiantes de bachillerato hacen referencia a las transferencias alimentarias con gran precisión, particularmente si se trata de reconocer los efectos que sobre una población presa tienen las variaciones de la población depredadora. Es decir, las relaciones ali- 
mentarias parecen aseguradas cuando se plantea seguir peldaño a peldaño los sucesivos cambios en una cadena alimentaria.

- Sin embargo, la nula o escasa alusión a otras fuentes de alimento (categoría A), a la hora de analizar el problema planteado, nos invita a pensar que, precisamente por la rigidez de la propia idea de "cadena", los estudiantes inciden en la unión indisoluble de los componentes como si de una cadena real se tratara.

- Otra concepción derivada de las respuestas encuadradas en la categoría B aparece nítidamente marcada: la identificación de eslabón con individuo (no población). En tales situaciones los alumnos expresan la idea de catastrofismo asociado a la depredación. Peterfalvi y otros (1987) señalan que las figuras y los ejemplos con que se suelen representar las cadenas tróficas en los textos (y es también el caso de la cuestión del problema) refuerzan la idea de fragilidad de la cadena y de sus componentes.

- En los comentarios de la categoría C aparece representada la idea opuesta: el régimen alimentario está desprovisto de factores de determinación. Éste dependerá de las preferencias individuales. Así, si las presas desaparecieran, un depredador cualquiera se adaptaría a otro tipo de comida si quisiera. El modelo antropocéntrico parece impregnar esta concepción.

- En algunos casos no se establece diferenciación entre los organismos componentes de la secuencia alimentaria. La cadena trófica se presenta como una asociación de seres cuyo fin está marcado por los individuos más fuertes, más poderosos, depredadores de todos los demás. En esta concepción el principio de la cadena puede ser iniciado por cualquier individuo más pequeño que los posteriores.

\section{Otras concepciones}

Otras cuestiones analizadas nos han permitido reconocer también la manera de interpretar los estudiantes la influencia recíproca entre los componentes abioticos y el mundo vivo.

El aspecto más notable del valor atribuido a esta interdependencia es que las sustancias o componentes del entorno (agua, luz, etc.) no son consideradas con carácter limitante, ni tampoco perturbadas o modificadas por influencia de los factores bióticos, incluida la especie humana.

Generalmente, los estudiantes de bachillerato estiman que ciertas sustancias, como el agua,la luz del sol, son imprescindibles para la vida. Sin embargo, no se relaciona su importancia con la intervención de dichos componentes en la formación de estructuras de los seres vivos. A este respecto, al analizar la forma de nutrición vegetal, la expresion «se alimenta de tierra» cobra todo su sentido cuando las alumnas y alumnos establecen una identificación absoluta entre abonos, tierra, etc., y alimento de las plantas. Investigaciones anteriores como las realizadas por Wandersee (1983), Bell (1984, 1985), Barker y Carr (1989) o Gené (1989), etc. inciden en dicha idea.

Como consecuencia de los resultados obtenidos consideramos que a la hora de proponer el tipo de contenidos y actividades para el estudio de la ecología se habrá de tener en cuenta, entre otras cosas, las ideas de los estudiantes. El hecho de que las representaciones comentadas se mantengan entre estudiantes de cursos superiores (alumnos de Biológicas incluso) nos invita a considerar que las causas de esta situación -algunas de las causas al menos- arraigan en el modo de aprendizaje de estos conceptos, por lo cual comentaremos a continuación algunas de las estrategias propuestas para favorecer la superación de las dificultades que se acaban de resumir.

\section{CÓMO ABORDAR LAS DIFICULTADES PLANTEADAS}

Los comentarios siguientes se justifican a partir de un trabajo desarrollado con varios grupos de estudiantes de $1^{9}$ de BUP ( $2^{\mathrm{g}}$ ciclo de la actual secundaria obligatoria) con los que a Io largo de los cursos 1988-91 se fueron definiendo el tipo de contenidos y las actividades más relevantes para la educación ambiental. Dicho trabajo nos permite señalar que a la hora de iniciar los estudios de ecología con alumnos del actual $2^{s}$ ciclo de secundaria deben planificarse actuaciones que comprendan, al menos, los siguientes aspectos:

- Qué ecología estudiar y donde realizar estudios de ecología para facilitar la educación ambiental.

-Qué estrategias de aprendizaje utilizar para superar las concepciones iniciales de los estudiantes.

\section{Qué ecología estudiar y dónde realizar estudios de ecologia}

En los últimos años han empezado a surgir algunas propuestas sobre el tipo y número de conceptos que debería incluir un curso de ecología para alcanzar con éxito la comprensión de principios ecológicos enfocados a la educación ambiental (Hungerford y Peyton, 1992; Giordan y otros 1993; García, 1994). También la British Ecological Society elaboró en 1987, a través del Dr. Malcolm Cherret, un cuestionario con una lista de 50 conceptos dirigido a los miembros de la sociedad con el fin de determinar cuáles eran los conceptos que los ecólogos consideraban de mayor importancia para la enseñanza de la ecología. Los resultados publicados por Cherret (1989) no muestran muchos acuerdos en la ordenación de los mismos, pero las nociones de ecosistema, flujo de energía, ciclo de los elementos, redes alimentarias, diversidad de especies, bioacumulación en cadenas alimentarias y niveles tróficos parecen gozar, por la clasificación establecida, de cierto consenso entre los especialistas. Tal elección concuerda con una de las 
tradiciones del pensamiento ecológico: la que remarca la importancia de los ecosistemas como entidades de estudio que permiten entender la interpenetración mutua de los aspectos físicos y de los bióticos. Del mismo modo, Gagliardi (1991) señala que los obstáculos mayores para el pensamiento del entorno son cómo construir y utilizar conceptos que permitan pensar globalmente; en tal sentioo expone que conceptos estructurantes sobre el significado de ecosistema u otros sistemas del entorno pueden ser, por ejemplo, autoconstrucción de organismos, átomos, moléculas, transferencia de materia y energía, población, microorganismos y factores limitantes.

Uno de los elementos de la teoría ecológica, que nos parece más interesante đesde el punto de vista del planteamiento global del ecosistema, es que muestra la inseparabilidad del entorno físico y los factores bióticos y, en expresión de Levins y Lewontin (1980), interpreta tanto los efectos de un elemento o población hacia muchos (elementos o poblaciones) como los efectos de muchos hacia uno. Esta idea constituye uno de los pilares fundamentales de este estudio, del que parte la búsqueda de una conexión con la educación ambiental de los estudiantes.

Pero el contenido de esta orientación, tan complejo en algunas de sus formulaciones, puede enmascarar la perspectiva ambiental si no se eligen los principios y métodos adecuados para su estudio. Como dicen Adams y otros (1985), la mayoría de la ciencia que se enseña no se relaciona con los problemas que existen en la sociedad, pues hay con frecuencia un mal emparejamiento entre la ciencia que se enseña y la que se necesita. A la hora de enfrentarnos con el tipo de conceptos y principios de ecología que se deben incluir en un corto curso dedicado a estudiantes de secundaria es preciso formular los objetivos de manera que permitan ampliar progresivamente los conocimientos y competencias para actuar de forma comprometida con la defensa del medio. Los contenidos que se aborden con los estudiantes deben ser tales que faciliten la labor de integración de significados y ayuden a tomar decisiones sobre problemas ambientales.

Los conceptos y principios seleccionados para este trabajo se fundamentan en las ideas arriba expuestas. Planteamos que se presente el concepto de ecosistema como concepto integrador de todos los demás, ya que, como nos recuerdan Giordan y otros (1988), sin ser la noción central de la ecología científica, el concepto de tcosis. tema está, cuando menos, profundamente inserto en la red de conceptos, teorías e hipótesis de esta disciplina. Un ecosistema concreto (laguna de agua dulce) es el marco de referencia para el desarrollo de los demás conceptos de ecología proptuestos para este estudio. La esperada transferencia de los conocimientos derivados de dicho aprendizaje hacia la educación ambiental se resume en los postulados que muy sintéticamente recogemos para apoyar la selecclón de conceptos y principios de ecología siguientes:

- Estudio de factores abióticos del ecosistema. Nuestra posición en la biosfera es la de useres ecodependientes», deudores de la doble identidad que impone la relación con los seres humanos y con el resto de los componentes de los ecosistemas, también los abióticos (Morin, 1984). Se trata de que los estudiantes sepan identificar algunos de estos componentes susceptibles de modificación por la actividad de los seres vivos, incluida la actividad humana.

- Estudio de componentes bióticos. Proponemos analizar un conjunto limitado de organismos. Aprovechando la fascinación que los estudiantes de los primeros cursos de bachillerato sienten hacia los seres vivos y hacia el empleo de aparatos para su observación, nos basamos en la identificación đe organismos microscópicos de agua dulce como vía de aproximación a la diversidad. La identificación de organismos autótrofos y heterótrofos y sus características celulares permitirá modificar alguna de las concepciones anteriormente comentadas y reconocer la variedad de organismos fotosintetizadores.

- Incorporación de materia y energía por los organismos autótrofos. Con base en la producción primaria vegetal se edifica la estructura piramidal $[. .$.$] , con sus herbivo-$ ros, sus depredadores, hasta la cúspide de la piramide, formada por los superdepredadores (Acot, 1990). Se trata de que los estudiantes analicen la importancia de los productores en toda secuencia trófica. Para ello, proponemos la elaboración de cadenas tróficas, previa información de los hábitos alimentarios de los organismos reconocidos.

- Transferencia de materia y energía a través de consumidores: análisis de redes alimentarias y niveles trófi$\cos$. El estudio de redes alimentarias es un excelente camino para que los estudiantes tomen conciencia del entorno natural, es decir, que se den cuenta tanto de las partes individuales (organismos) como de que esas partes contribuyen a formar el conjunto (ecosistema) (Alexander, 1982). Se proponen problemas para reconocer los cambios que sufre una población de la red ante las variaciones bruscas de otra población. La identificación de las redes utilizadas como base de este estudio y el tipo de problemas planteados se pueden encontrar en los anexos III y IV.

- Papel de los descomponedores y ciclo de los nutrientes. Si las plantas y los consumidores no sufrieran finalmente la descomposición, el suministro de nutrientes se agotaría y la vida desaparecería de la Tierra (Begon, Harper y Townsend, 1988). La contribución de los descomponedores, tan poco reconocida (Margalef, 1991), aporta nuevos datos a la comprensión global del ecosistema si se integra su actuación en el ciclo de los nutrientes.

- Influencia humana en los ecosistemas. La reducción de la diversidad en los ecosistemas es uno de los efectos más preocupantes de nuestra intervención en la biosfera (García Novo, 1992). Se incluirá, pues, la toma de muestras y el análisis de un medio perturbado.

Como se viene sugiriendo, el estudio de estos principios se ha basado en los elementos que proporciona un ecosistema concreto. ¿Dónde, pues, realizar los estudios de 
ecología? En cualquier curso de ecología, dice Gray (1982), es vital que se incluya suficiente trabajo de campo para que los estudiantes puedan tener experiencias del hábitat estudiado y, al mismo tiempo, puedan aprender las destrezas básicas implicadas en los muestreos y datos.

Una situación ideal sería aquélla en la que los profesores contáramos con lugares que pudieran ser fácilmente frecuentados y visitados. Sin embargo, esta aspiración no deja de ser utópica, al menos para aquellos centros cuyo emplazamiento se localiza en distritos industrializados, lejos de áreas naturales de fácil acceso.

La importancia del entorno local radica, esencialmente, en su carácter familiar para profesores y alumnos, y además por ofrecer la oportunidad de continuas y progresivas observaciones de fenómenos naturales. A pesar de que para algunos autores (Turner, 1988) un área natural puede parecer aplastante en su complejidad, se puede utilizar con éxito si se delimitan los elementos que se van a tener en cuenta para la realización del trabajo programado. Para un estudio como el anteriormente propuesto recomendamos que se tengan en cuenta los aspectos siguientes:

- Seleccionar un área de fácil acceso con el fin de poder realizar traslados con los alumnos para la toma de muestras que completen el trabajo de aula y laboratorio.

- Emplear en el estudio un pequeño conjunto de buenas plantas y buenos animales que contribuyan a clarificar los principios ecológicos. Para analizar las relaciones tróficas, por ejemplo, es necesario determinar con qué grupos de organismos se pueden elaborar entramados de redes bien definidas.

- Reconocer los componentes del ecosistema en la estación en que se realice la salida. Preparar claves sencillas de identificación que señalen los rasgos más sobresalientes de las especies con las que se desea contar para el estudio que se propone. Las alumnas y alumnos de secundaria pueden utilizar claves cuando el ámbito en el que tienen que tomar decisiones se refiere a pocos organismos con características obvias y a condición de que las dicotomías se presenten con reglas bien definidas. Como señalan Shayer y Adey (1984), los estudiantes del nivel que estamos comentando comienzan a usar reglas de clasifićcación como inclusión en una clase, pero no pueden emplear las «claves" de expertos ya que éstas se basan en estrategias que incluyen a todo el conjunto de seres descritos y el alumno busca características definitorias de cada organismo; por ejemplo, los insectos tienen seis patas.

- Definir una zona en que aparezca clara variación de comunidades vegetales y animales afectadas por la variación de componentes abióticos. Por ejemplo, en un ecosistema de agua dulce reconocer la presencia de algas verde-azuladas en lugares con abundantes nutrientes.

- Utilizar instrumentos de observación y de medida sencillos y sólo los necesarios para la obtención de los datos que se analizarán en el aula o laboratorio. Como señala Humphreys (1987), si el trabajo de campo es el medio para aclarar conceptos, debe tenerse en cuenta que gran parte de actividad asociada al uso de equipos puede ser un factor de distracción.

\section{Cómo afrontar las dificultades de aprendizaje: breves consideraciones didácticas}

La investigación en didáctica de las ciencias ha aportado ya algunas conclusiones importantes a la hora de organizar las actividades para intervenir en el cambio o evolución de las ideas de los alumnos como las anteriormente comentadas. A la hora de planificar el trabajo de clase y de seleccionar la forma de intervención adecuada no hemos olvidado las respuestas aportadas por otras investigaciones, como las que inciden en el cambio o la evolución de las concepciones iniciales. El modelo de aprendizaje como cambio conceptual fue expuesto por primera vez en varios trabajos aparecidos a principios de los ochenta (Hewson, 1981; Posner et al., 1982; Osborne y Wittrock, 1983). El aspecto central del modelo, según Solís (1984), es el de analizar el proceso de enseñanza/ aprendizaje como una interacción entre las ideas previas del alumno y la información nueva. Las estrategias de aprendizaje que parten de la importancia de tener en cuenta las concepciones de los estudiantes incluyen actividades varias, encaminadas a cambiar las ideas iniciales 0 a ampliar el significado de las mismas. (Remitimos a la ya abundante bibliografía sobre el aprendizaje como cambio conceptual. Detalladas revisiones de esta perspectiva aparecen en los trabajos de Jiménez, (1991) y Gil (1993).

También hemos recurrido a experiencias suficientemente contrastadas de otros modelos de aprendizaje basados en el asociacionismo. Así hay que entender nuestro interés por ampliar el significado de cadena alimentaria mediante la resolución de problemas de complejidad creciente en redes alimentarias. Con este tipo de actividades, los autores (Griffitbs y Grant, 1985, en el anexo IV) han validado nueve jerarquías de aprendizaje del concepto de red, según la capacidad de los estudiantes para resolver la manera en que una población es afectada por las variaciones bruscas de otra. Se espera obtener mejor comprensión del concepto a medida que las respuestas incluyan mayor número de poblaciones y más vías de análisis.

Queremos señalar con ello que la adopción de estrategias basadas en un único modelo de aprendizaje puede que no sea el camino para introducir todos los conceptos implicados en el tema. Quizás debamos considerar, como señala Pozo (1989), que los cambios en las concepciones de Ios estudiantes se pueden producir tanto por asociacionismo como por reestrưcturación.

Según los supuestos arriba comentados, la unidad incluye distintas formas de plantear los trabajos de los estudiantes. Mediante algunas de las actividades se trata de hacer que las alumnas y alumnos perciban la diferencia 
entre sus concepciones iniciales y los resultados de Ia observación o experimentación (reconocimiento de características celulares de organismos autótrofos y heterótrofos, por ejemplo); en otras situaciones se trata de introducir nuevos conceptos y principios (ciclo de la matería y energia); o se proponen experiencias y problemas para ampliar las concepciones iniciales (caso del análisis de los cambios en las poblaciones de la red alimentaria); o bien se intenta poner a prueba sus conocimientos en situaciones variadas para mostrar el poder explicativo de sus predicciones; etc.

\section{PROBLEMA PLANTEADO. DISEÑO DE LA INVESTIGACIÓN}

Las actividades anteriormente comentadas se fueron definiendo a lo largo del trabajo de varios años con grupos de estudiantes de $1^{9}$ de BUP. Con el fin de determinar de qué manera influye el trabajo de campo propuesto en la comprensión de los principios de ecología y si el conocimiento adquirido se transfiere o no en la resolución de un problema ambiental, se realizó una investigación (Fernández Manzanal, 1993) con un diseno experimental de dos grupos $(N=67)$ que adoptó la forma siguiente:

Donde la salida al campo representa la exposición del número de grupos a la variable independiente o tratamiento. En dicha salida los estudiantes del grupo experimental observaron las características del lugar, reali- sentido que se viene señalando, es decir, que se facilitaría la asimilación de conocimientos de ecología utilizando como apoyo un ecosistema concreto del entorno, y que éste, visitado y conocido, surgirá como referente de los argumentos empleados en la evaluación de un problema ambiental.

Se eligió un diseño de dos grupos naturales de $1^{\circ}$ de BUP, uno experimental y otro de control, por ser uno de los diseños experimentales más característicos de la inves. tigación en educación, al ser los grupos entidades formadas naturalmente (Campbell y Stanley, 1988).

El pretest permitió asegurar Ia equivalencia de ambos grupos en todos los conceptos analizados. Como señalan Campbell y Stanley, cuanto más similares sean en su reclutamiento el grupo experimental y el de control y más se confirme esa similitud por los puntajes del pretest, más eficaz resulta ese control.

En el desarrolio de Ia investigación se extremaron las precauciones sobre la historia intrasesional mediante el seguimiento pormenorizado de todas las actividades y su anotación en el diario de sesiones de clase. A tal efecto, las variaciones introducidas en uno de los grupos ante sugerencias de los alumnos, no previstas en la experimentación, fueron trasladadas al otro grupo con el fin de que ambos recibieran la misma información.

Con el fin de de controlar los factores que pudieran atentar a la validex interna del diseño, se hizo un detalla. do estudio tanto de las características de los grupos que constituyeron la muestra como de las condiciones en las

Tabla I

Características del diseño experimental. Adaptado de Jiménez, López-Barajas y Pérez (1983).

\begin{tabular}{|c|c|c|c|c|c|}
\hline \multicolumn{2}{|c|}{ Astignación } & \multirow[t]{2}{*}{ Grupo } & \multirow{2}{*}{$\begin{array}{c}\text { Situación } \\
\text { inicial }\end{array}$} & \multirow[t]{2}{*}{ Tratamiento } & \multirow{2}{*}{$\begin{array}{c}\text { Variable } \\
\text { dependiente }\end{array}$} \\
\hline Sujetos & Tratamiento & & & & \\
\hline No azar & Azar & Experimental & $o_{1}$ & Salida campo & $\mathrm{O}_{3}$ \\
\hline No azar & Azar & Control & $\mathrm{O}_{3}$ & & $\mathrm{O}_{4}$ \\
\hline
\end{tabular}

zaron una descripción del entorno, clasificaron las especies vegetales circundantes, describieron el hábitat de algunas especies de aves, midieron la temperatura y la turbidez del agua en distintas zonas, registraron los canales de alimentación, tomaron muestras de plancton para su observación microscópica y clasificación, etc. Los estudiantes del grupo de control realizaron las mismas actividades de aula y de laboratorio, en el mismo tiempo y con los mismos componentes pero no obtuvie" ron las muestras ni participaron en el trabajo de campo.

Partíamos de la idea, ya mostrada por Kinsey (1984), de la conexión existente entre el dominio cognitivo y afectivo, idea que nos permitió definir la investigación en el que se realizó la experiencia; no sólo en lo que se refiere a la historia intrasesional citada, sino en lo que atañe a la maduración, administración de tests, instrumentación, regresión, selección, etc.

Respecto a los instrumentos de toma de datos, se utilizaron técnicas cualitativas y cuantitavas de análisis, ya que coincidimos con Jiménez (1989) en que los dos tipos de técnicas suministran datos complementarios y proporcionan mayor riqueza en un estudio que se ocupa de ambas dimensiones. Como señala Caride (1993), los instrumentos cuantitativos son muy interesantes por lo que suponen de «reducción a medida» de la información disponible, con capacidad para un tratamiento analíti- 
co en términos estadísticos e informáticos; los instrumentos cualitativos, a su vez, tienen interés por lo que implican de «apertura a contingencia» manteniendo el carácter comprensivo e interpretativo de lo que es o sucede.

Para definir la situación inicial (datos $\mathrm{O}_{1}$ y $\mathrm{O}_{3}$ del diseño) se utilizó un pretest y los comentarios derivados de la observación del trabajo de clase durante el desarrollo del tema. Para definir los resultados de la variable dependiente: evolución de conceptos preliminares, componentes y relaciones entre los componentes de un ecosistema y transferencia a la educación ambiental (datos $\mathrm{O}$, y $\mathrm{O}_{4}$ del diseño), se utilizó un postest, un mapa conceptual y entrevistas semiestructuradas. La valoración del mapa conceptual se hizo atendiendo al esquema propuesto por Novak (Novak y Gowin, 1988), asignando puntuaciones a cada una de las seis categorías establecidas, lo cual permitió descubrir la forma de organizar en los estudiantes los conceptos de ecología y el tipo de relaciones que entre ellos establecen. Las entrevistas fueron objeto también de un pormenorizado análisis con el fin de descubrir la transferencia de los conocimientos a la resolución de un problema ambiental. En la valoración de los resultados se realizó un estudio cualitativo basado en las recomendaciones de Taylor y Bogdan (1986) sobre la manera de trabajar con los datos. Se buscaron diferencias entre el grupo experimental y de control en función del lenguaje empleado y del análisis de las proposiciones y explicaciones que daban los estudiantes cuando se enfrentaban a un problema de perturbación del entorno enunciado del modo siguiente:

Imagina que te encargan del cuidado de un espacio natural como un parque o la laguna que hemos estudia. do. A la hora de hablar de su buen o mal funcionamiento, ¿qué aspectos tendrias en cuenta?, ¿en qué te fijarias? $S i$ de repente encontraras que han muerto muchos individuos de la misma especie, ¿qué pensarias?

Para su valoración se tuvieron en cuenta tanto los conocimientos puestos en juego como las soluciones aportadas para controlar la situación problema. De este modo se elaboraron seis tablas para cada grupo con los resultados derivados de la interpretación que los estudiantes hicieron, atendiendo a los conceptos puestos en juego, la clasificación de los mismos (tipologías) y la identifica. ción de los comentarios que hacían referencia expresa a las causas del problema y a su solución. De este modo, se trataron de encontrar discrepancias entre los alumnos en función del conjunto de supuestos, conceptos y proposiciones interrelacionados que configuraban, a nues. tro parecer, una visión del mundo: su particular visión del problema.

\section{Tratamiento estadistico einterpretación de los resultados}

Tanto para el análisis del pretest como del postest y del mapa conceptual, se recurrió a pruebas no paramétriças. Esto nos evitó hacer asunciones acerca del cumplimiento de los supuestos del modelo paramétrico, en particular, los que se refieren a la normalidad de la población y a la medición de variables (Siegel, 1990). El nivel de medida aplicado fue el ordinal fío.

La prueba utilizada para medir las diferencias entre el grupo experimental y de control (en cada cuestión del pretest, del postest y en el mapa conceptual) fue la $U$ de Mann Whitney, que es la más potente para el caso que aquí nos ocupa, de dos grupos independientes. La valoración estadística se realizó con todos los conceptos y relaciones exploradas en las cuestiones y se aplicó, asimismo, a las medidas numéricas del mapa conceptual. A la prueba aplicada se asignó un nivel de confianza del $95 \%$, o lo que es lo mismo, un nivel de significación $\alpha=0,05$. Para establecer el contraste entre el aprendizaje de conceptos de ecología antes y después de la instruc. ción, se eligió, de nuevo, una prueba no paramétrica. En este caso la aplicable a una medida ordinal al nivel más preciso y fino: la $T$ de Wilcoxon o de pares igualados, a la que se asignó un nivel de confianza del $99 \%$, o lo que es lo mismo, un nivel de significación $\alpha=0,01$. El cálculo se realizó mediante la aplicación del programa Nonparametric Tests, del paquete estadístico NCSS (Number Cruncher Statistical System, versión 5.01), de J.L. Hinze.

\section{RESULTADOS DE LA INVESTIGACIÓN}

Después del estudio del tema y de la aplicación del tratamiento resumimos las siguientes diferencias en la variable dependiente (Tabla II).

\section{Conceptos preliminares}

El primer aspecto tiene que ver con la consideración de conceptos preliminares: concepciones de animal y vegetal, nutrición vegetal. En estos conceptos tanto los alum. nos y las alumnas del grupo experimental como los del grupo de control muestran diferencias significativas con Ia situación de partida ( $\mathrm{T}$ de Wilcoxon). Ambos grupos adquirieron además el mismo tipo de aprendizaje. No se encontraron diferencias significativas en la aplicación de la $U$ de Mann-Whitney. Consideramos, en consecuencia, que las actividades de aula y laboratorio contribuyeron a facilitar la ampliación de las ideas iniciales sobre el significado de animal/vegetal o nutrición vegetal.

\section{Conceptos de ecología}

En cuanto al aprendizaje de nuevos conceptos de ecología, los datos obtenidos revelan que la mayoría de los estudiantes de los dos grupos adquirieron el conocimiento declarativo adecuado para clasificar y diferenciar los niveles tróficos del ecosistema. Los datos obte. nidos revelan que una gran proporcion de estudiantes identificaron los niveles mediante el empleo correcto de términos de la teoría ecológica. En este apartado no aparecieron diferencias significativas entre los grupos de la muestra. 
Tabla II

Resumen de los resultados de los conceptos y principios de ecología explorados. Se señalan con * los datos que muestran diferencias significativas. (M.C.: Valoración del mapa conceptual).

\begin{tabular}{|c|c|c|c|c|}
\hline & & \multicolumn{2}{|c|}{ T de Wilcoxon } & \multirow[t]{2}{*}{ U de Mann-Whitney } \\
\hline & & Grupo experimental & Grupo de control & \\
\hline \multirow[t]{3}{*}{ Conceptos preliminares } & Significado animal & $0.0001^{*}$ & $0.0001^{*}$ & 0.602 \\
\hline & Significado vegetal & $0.0001^{*}$ & $0.0001^{*}$ & 0.231 \\
\hline & Nutrición vegetal & $0.0000^{*}$ & $0.0000^{*}$ & 0.295 \\
\hline \multirow[t]{8}{*}{ Conceptos de ecología } & \multicolumn{3}{|c|}{ Identificación de niveles tróficos } & 0.915 \\
\hline & \multicolumn{3}{|c|}{ Clasificación de los componentes del ecosistema (M.C.) } & 0.256 \\
\hline & \multicolumn{3}{|c|}{ Relaciones troficas (nivel 6) } & 0.930 \\
\hline & \multicolumn{3}{|c|}{ Relaciones tróficas (nivel 9) } & $0.009^{*}$ \\
\hline & \multicolumn{3}{|c|}{ Ciclo de los elementos } & $0.025^{*}$ \\
\hline & \multicolumn{3}{|c|}{ Relaciones entre componentes del ecosistema (M.C.) } & $0.045^{*}$ \\
\hline & \multicolumn{3}{|c|}{ Organización jerárquica de conceptos (M.C.) } & $0.002^{*}$ \\
\hline & \multicolumn{3}{|c|}{ Significado de ecosistema (M.C.) } & $0.030^{*}$ \\
\hline
\end{tabular}

Lo mismo puede decirse de la identificación de los organismos componentes de los niveles señalados. El conocimiento y la comprensión parecen asegurados al expresar en cada caso ejemplos correctos en distintos ecosistemas, no sólo en el estudiado. Tampoco en este aspecto encontramos diferencias significativas entre los grupos.

En el análisis de las relaciones tróficas (primer problema del anexo IV) no aparecieron diferencias significativas cuando se trataba de valorar las implicaciones que el cambio de una población produce en otras que se representan en un entremado simple, semejante al de cadena alimentaria. En este caso, se pueden deducir los cambios en otras poblaciones siguiendo escalón a escalón la secuencia representada. El modelo, califícado con la numeración de 6 atendiendo a las destrezas implicadas, según la clasificación de Griffiths y Grant (1985), no muestra diferencias significativas entre los grupos.

Aparecen diferencias significativas entre el grupo experimental y de control al analizar las relaciones entre los componentes; tanto entre los componentes bióticos como entre los bióticos y abióticos. Así:

- Los estudiantes del grupo experimental muestran mayor destreza para reconocer vías entrecruzadas por las que diversas poblaciones son afectadas ante la variación de otra. En el análisis del segundo problema de redes planteado -situado en la calificación 9 de Griffiths y Grantm aparecen diferencias significativas entre los dos grupos.
- También la relación entre los componentes bióticos y abióticos del ecosistema ha sido mejor comprendida por los estudiantes del grupo experimental. El seguimiento del ciclo de los elementos y la importancia de los mismos muestran diferencias significativas entre los dos grupos.

- Los estudiantes del grupo experimental establecen mayor número de conexiones entre los componentes del ecosistema, mediante explicaciones de la relación entre unos y otros componentes. La valoración del mapa conceptual, que expresa las relaciones entre los conceptos con la explicación de dicha relación, es una prueba de. ello.

- Los estudiantes que realizan el trabajo de campo expresan de forma significativamente mejor la organización de los conceptos de ecología. Así entendemos que este apartado de valoración del mapa muestre claras diferencias entre los dos grupos, favorables al grupo experimental.

- Finalmente, en cuanto al significado mismo de ecosistema como expresión y sintesis de los conocimientos adquiridos, también los mapas muestran difencias significativas, favorables, de nuevo, al grupo experimental.

\section{Transferencia de conocimientos de ecología}

En cuanto a la traducción de los conocimientos en la sensibilización hacia el cuidado de los ecosistemas y la 
educación ambiental, el análisis de las entrevistas mostró diferencias favorables hacia el grupo experimental en todos los aspectos que se analizaron para la valoración del problema propuesto. En los comentarios de los estudiantes de ambos grupos se identificaron seis temas: análisis del agua, análisis de los organismos, análisis del entorno, influencia de la intervención humana, análisis de la tierra y el aire, y términos de la teoría ecológica aplicados en la resolución del problema. Todas las diferencias encontradas se manifestaron tanto en la variedad de conceptos empleados como en la clasificación de los aspectos que habría que considerar (tipologías en la terminología de Taylor y Bogdan, 1986) o en las expresiones (proposiciones) que los estudiantes utilizaban para caracterizar la perturbación o la forma de resolver la situación problemática. Señalamos las siguientes diferencias:

- La importancia que los alumnos del grupo experimental atribuyen a los componentes abióticos en la valoración del estado del ecosistema. En el problema que nos ocupa, el interés hacia dichos componentes se expresa al señalar que lo más importante es conocer el tipo de sustancias que producen variaciones y al contemplar en la descripción varios de los factores analizados en la salida (los estudiantes del grupo de control no señalan estos factores).

- La necesidad de medida o, en general, de hacer análisis exhaustivos de los componentes no sólo del medio abiótico sino de los seres vivos del ecosistema es una constante que se reproduce en los comentarios de los alumnos del grupo experimental. A la hora de estudiar las variaciones de los componentes bióticos, tal idea se manifiesta también mediante la importancia del recuento del número de individuos y el conocimiento de los hábitos de cada especie. Señalan la necesidad de informarse sobre las características de especies concretas para reconocer mejor la perturbación del caso problema.

- Si en el diálogo se introduce la posibilidad de cambios en el número de individuos de una población, la interpretación de los mismos es distinta en cada grupo. Mientras que para los estudiantes del grupo de control tales cambios se presuponen siempre como descenso del número de individuos (como consecuencia de las variaciones en la población depredadora), los estudiantes del grupo experimental consideran la posibilidad de que ante las modificaciones del medio algunas especies pueden aumentar en número considerablemente.

- Los estudiantes del grupo experimental hacen constantes referencias a las relaciones entre diversos componentes del ecosistema estudiado, en particular en lo que se refiere a la intervención humana. Cuando se trata este tema recurren a múltiples datos de los rastros de dicha influencia en este ecosistema, remarcando las derivaciones que, a largo plazo, pueden concurrir en la modificación de especies y del entorno. En tal sentido proponen formas de control que tienen que ver tanto con el comportamiento individual como con sistemas de regulación y normas administrativas.
Ante problemas concretos, como los enunciados, los estudiantes del grupo que han visitado el ecosistema utilizan un lenguaje más preciso y, lo que es más importante, señalan los caminos de intervención para evitar situaciones semejantes. En nuestra opinión, si las alumnas y alumnos emplean términos de Ia teoría ecológica para enfrentarse a problemas ambientales y si tales conocimientos influyen en la manera de buscar soluciones a los mismos, el aprendizaje de principios de ecología se configura como un importante objetivo de la educación ambiental.

\section{CONCLUSIONES}

Como se acaba de resumir, parece clara la existencia de un cambio notable en los conceptos preliminares respecto a la situación inicial. Concluimos, por tanto, que las estrategias de intercambio diseñadas han sido eficaces a la hora de producir la evolución de las ideas previas de los y las estudiantes hacia nociones más adecuadas desde el punto de vista científico.

El aprendizaje de los componentes de los ecosistemas, de su función -identificación de niveles-, y, en especial, lo que atañe a la función productora de los vegetales nos parece de gran importancia por su repercusión en el cuidado del medio y en la educación ambiental.

En ambos tipos de conceptos los estudiantes de la muestra han manifestado una comprensión semejante. Utilizando los criterios de Klausmeier (1976) para definir el aprendizaje de conceptos, se podría concluir que la mayoría de los alumnos de esta experiencia han pasado del nivel clasificatorio (generalización y constatación de la semejanza de dos o más objetos) al nivel formai, al ser capaces de definir los conceptos en función de determinados atributos, en este caso su función en los ecosistemas.

Por otra parte, los alumnos y alumnas que han realizado el trabajo de campo:

- Expresan con mayor precisión la importancia de los componentes abióticos y su incidencia en el mantenimiento de los seres vivos, y señalan a la vez cuáles son los factores más importantes de degradación en el ecosistema elegido.

- Resuelven los problemas más complejos de relaciones troficas comentados. Ello indica que perciben con mejor claridad la variedad de caminos entrecruzados entre los organismos del ecosistema y las relaciones entre los mismos.

- Aprenden a expresar el significado de ecosistema incluyendo no sólo las partes sino cómo se articulan para su funcionamiento. Se podria decir que adquieren más destrezas para realizar la síntesis de información y son más capaces de organizar los elementos y ordenarlos en la constitución de un cuadro complejo. 
- Analizan un problema ambiental teniendo en cuenta tanto los elementos reconocidos en las salidas al campo como argumentos de la teoría ecológica que se apoyan en los datos derivados del trabajo de campo y del tratamiento de los mismos en el estudio del tema.

Como valoración final de los resultados arriba comentados nos interesa señalar que los estudiantes que analizan los componentes e interacciones del ecosistema a partir del estudio del lugat, de la toma de muestras; de la observación in situ de las especies, etc. reconocen mayor cantidad de componentes y adquieren mejor comprensión de las relaciones, probablemente porque tienen un referente en el que situar los análisis que se van realizando en el aula o laboratorio.

Quizás este tipo de trabajo de campo contribuye a la educación ambiental tanto por las actividades especialmente planificadas como por el efecto secundario de las mismas. Y un efecto secundario es que el espacio visitado aparece no sólo como «algo visto» sino que una vez que las características ban sido descritas y aclaradas se muestra como «algo sentido». Se trata, como indica Caduto (1992), no sólo de aumentar los conocimientos de los estudiantes sino de lograr involucrarles emocionalmente a través de experiencias prácticas que puedan influir en su predisposición hacia la preservación del medio mediante el compromiso y la acción.

En nuestra opinión, la educación en los valores de defensa del medio ambiente no debe consistir en un

\section{REFERENCIAS BIBLOGRÁFICAS}

ACOT, P. (1990). Cómo nació la ecología. Mundo científico, 98(10), pp. 70-77.

ADAMS, C.E., CHARLES, C., GREEN, J. y SWAN, M. (1985). New designs in conservation ecology education. The American Biology Teacher, 47(8), pp. 463-469.

ADENIYI, E.O. (1985). Misconceptions of selected ecological concepts held by some Nigerian students. Journal of Biological Education, 19 (4), pp. 311-316.

AKINSOLA, P.(1990). Attaining meaningful learning of concepts in genetics and ecology: An examination of the potency of the concept-mapping technique. Journal of Restarch in Science Teaching, 27(5), pp. 493-504.

ALEXANDER, S.K. (1982). Food web analysis: An ecosystem approach. The American Biology Teacher, 44 (3), pp. 186, $189-190$. conjunto de códigos impuestos sino que debe nacer de la comprensión del significado que adquieren las alteraciones provocadas en los ecosistemas. Indudablemente este conocimiento debe ir acompañado del valor especial que, por su intensidad, tienen en nuestra época dichas alteraciones. Los estudios de ecología vendrían así a confluir con los conocimientos de otras disciplinas en la comprensión de nuestro papel en la biosfera y en la extensión de los nuevos valores de la educación ambiental.

En cuanto a la posibilidad de generalización de los resultados, los estudios experimentales de campo presentan alguna ventaja respecto a otros estudios por su realización en un marco real, pero las conclusiones son siempre probabilísticas y deben tomarse con las precauciones correspondientes a dicha situación. Aunque en el momento de redactar estas líneas ya se ha repetido la experiencia con otros grupos que han realizado el trabajo de campo, con resultados semejantes, hay que tener en cuenta que el escenario de la clase es siempre complejo y sólo posteriores estudios en los que estén implicados estudiantes de otros países y contextos permitirán extender y generalizar las conclusiones expuestas.

\section{AGRADECIMIENTOS}

Agradecemos a los companeros de Ciencias Naturales del IB Hermanos D'Elhuyar de Logroño su colaboración en Ia laboriosa preparación de las salidas al campo y ia puesta en práctica de los materiales elaborados.
ASTOLFI, J.P. (1987). Approche didactique de quelques aspects du concept d'écosystème. Aster, 3, pp. 9-18.

BARKER, M.A. (1985). Food, Working Paper, 226. University of Waikato: Science Education Research Unit.

BARKER, M.A. y CARR, M. (1989). Photosynthesis - Can our pupils see the wood for the trees? Journal of Biological Education, 23 (1), pp. $41-44$.

BEGON, M., HARPER, J.L. y TOWNSEND, C.R. (1988). Ecologia. Individuos, poblaciones y comunidades. Barcelona: Omega.

BELL, B.F. (1981). When is an animal not an animal? Journal of Biological Education, 15(3), pp. 213-218.

BELL, B. F.(1984). Aspects of secondary students' understanding of plant nutrition: Summary report. University of Leeds: C.L.I.S.P. 
BELL, B.F. (1985). Student's ideas about plant nutrition: what are they? Journal of Biological Education, 19 (3), pp. 213218.

BELL, B.F. y BARKER, M.A. (1982). Towards a scientific concept of «animal». Journal of Biological Education, 16 (3), pp. 197-200.

BELL, B.F.y BROOK, A.(1984).Aspects of secondary students' understanding of plant nutrition: full report. University of Leeds: C.L.I.S.P.

BOOTH, P.R. (1979). The teaching of ecology in school (with one appendix by C.A. SINKER). Journal of Biological Education, 13 (4), pp. 261-266.

CADUTO, M.J., 1992, Guía para la enseñanza de los valores ambientales. Madrid: Los libros de la Catarata.

CAMPBELL,D. y STANLEY,J.(1988).Diseños experimentales y cuasiexperimentales en la investigación social. Buenos Aires: Amorrortu.

CARIDE, J.A. (1993). El análisis de contextos en educación ambiental. Máster en educación ambiental. Madrid: Fundación Universidad-Empresa/ UNED.

COLOM CAÑELLAS, A.J. (1993). Situación actual de la Educación Ambiental. Papers: Medio Ambiente y Educación. Barcelona: Fundación La Caixa.

CHERRETT, M. (1989). Key concepts: the results of a survey of our members' opinions, en Ecological Concepts, Cherrett, J.M. (ed.), pp. 1-16. Oxford: Blackwell.

FERNÁNDEZ MANZANAL, R. (1993). La ecología en la educacion ambiental: Influencia del trabajo de campo en el aprendizaje de conceptos y relaciones de ecología en el bachillerato. Santiago de Compostela: Tesis doctoral.

GAGLIARDI, R., MARTINAND, J.L. y SOUCHON, CH. (1991). Concepts majeurs et concepts structurants pour l'education à l'environement. Actes JIIES XIII.

GAYFORD, C.G. (1989). Science Education and the Environment. Education in Science, January, 22/23.

GARCÍA, J.E. (1994). El conocimiento escolar como proceso evolutivo: aplicación al conocimiento de nociones ecológicas. Investigación en la Escuela, 24, pp. 65-76.

GARCÍA NOVO, F. (1992). Perspectiva ecológica. Master en Educación Ambiental. Madrid: Fundación UniversidadEmpresa/UNED.

GENÉ, A. (1989). I si les plantes no mengen tẹra, què mengen les plantes? Actes del II Simposio Internacional sobre l'Ensenyament de les Ciències Naturals, pp. 206-243. Tarragona.

GIL, D. (1993). Contribución de la historia y de la filosofía de las ciencias al desarrolio de un modelo de enseñanza/ aprendizaje como investigación. Enseñanza de las Ciencias, 11(2), pp. 197-212.

GIORDAN, A. et al. (1988). Conceptos de biología 1, La respiración. Los microbios. El ecosistema. La neurona. Barcelona: MEC-Labor.

GIORDAN, A. et al. (1993). Educación ambiental: principios de enseñanza y aprendizaje. Bilbao: Los libros de la Catatata.

GRAY, N.F. (1982). The use of percolating filters in teaching ecology. Journal of Biological Education, 16(3), pp. 183-186.
GRIFFITHS, A.K. y GRANT, B.A.C. (1985). High school students' understanding of food web: identification of a learning hierarchy and related misconceptions. Journal of Research in Science Teaching, 22 (5), pp. 421-436.

HEWSON, P.(1981). A conceptual change approach to learning Science. European Journal Science Education, 3(4), pp. 383-396.

HUMPHREYS, T.J.(1987). The evaluation of fieldwork: concept elucidation by transects. Journal of Biological Education 21(1), pp. 28-34.

HUNGERFORD, H.R. y PEYTON, R.B.(1992). Como construir un programa de educación ambiental. Madrid: Los libros de la Catarata.

JIMÉNEZ, M.P. (1989). Los esquemas conceptuales sobre la selección natural: análisis y propuestas para un cambio conceptual. Madrid: Tesis doctoraI.

JIMÉNEZ, M.P. (1991). Cambio las ideas sobre el cambio biológico. Enseñanza de las Ciencias, 9 (3), pp. 248-256.

JIMÉNEZ, M.J. y LALIENA, L.(1992). Transversales: Educación Ambiental. Madrid: MEC.

JIMÉNEZ, C., LÓPEZ-BARAJAS, E. y PÉREZ, R. (1983). Pedagogía Experimental $I$. Madrid: UNED.

KINSEY, T.G. et al. (1984). The effects of environmental studies course on the defensibility of environmental attitudes. Journal of Research in Science Teaching, 21(7), pp. $675-$ 683.

KLAUSMEIER, H.J. (1976). Conceptual development during the school years, en Levin, J.R. y Allen, V.L. (eds.), Cognitive learning in children. Theories and strategies. Londres: Academic Press.

LEVINS, R y LEWONTIN, R.(1980). Dialectics and reductionism in ecology. Synthese, 43, pp. 47-78.

MANTLE, G. y HEATH, S. (1986). The use of urban spaces for environmental education. Journal of Biological Education, 20 (4), pp. 279-286.

MARGALEF, R. (1974). Ecología. Barcelona: Omega.

MARGALEF, R. (1991). Teoría de los sistemas ecologicos. Universitat de Barcelona.

MORIN, E. (1984). Conciencia con consecuencia. Barcelona: Antrophos.

NOVAK, J.D, y GOWIN, D.B. (1988). Aprendiendo a aprender. Barcelona: Martínez Roca.

OSBORNE, R. y WITTROCK, M. C. (1983). Leaming Science: a generative process, Science Education, 67(4), pp. 489-508.

PETERFALVI, B., RUMELHARD, G. y VERIN, A. (1987) Relatios alimentaires. Aster, 3, pp. 111 189 .

PETERS, R.H. (1991). A critique for ecology. Cambridge: Cambridge University Press.

POSNER, G. J., STRIKE, K. A., HEWSON, P. W. y GERTZOG W. A. (1982). Accomodation of a scientific conception: towards a theory of conceptual change. Science Education, 66 , pp. 211-227.

POZO, J.I. (1989). Teorías cognitivas del aprendizaje. Madrid: Morata.

SHAYER, M. y ADEY, PH. (1984). La ciencia de enseñar ciencias. Madrid: Narcea. 
SIEGEL, S. (1990). Estadística no paramétrica. México: Trillas.

SOLIS, R. (1984). Ideas intuitivas y aprendizaje de las ciencias. Enseñanza de las Ciencias, 2(2), pp. 83-89.

TAYLOR, S.J. y BOGDAN, R. (1986). Introducción a los métodos cualitativos de investigación. Buenos Aires: Paidós.

TERRADAS, J. (1979). Ecología y educación ambiental. Cuadernos de Biología. Barcelona: Omega.

TROWBRIDGE, J.E. y MINTZES, J.J. (1988). Alternative conceptions in animal classifications: a cross-age study. Journal of Research in Science Teaching, 25 (7), pp. 547. 571 .

TURNER, B. (1988). Applying ecology: domestic pests, a suitable case for treatment. Journal of Biological Education, 22(3), pp. 183.188.
UNESCO (1977). Conference intergouvernamentale sur l'education relative d̀ l'environnement, Tbilisi. París: Unesco.

VELASCO, J.M. (1991). ¿Cuándo un ser vivo puede ser considerado animal? Análisis de las concepciones altemativas del alumnado acerca del significado de «animal». Enseñanza de las Ciencias, 9 (1), pp. 43-52.

WANDERSEE, I. H. (1983). Students' misconceptions about photosynthesis: A cross age study. Paper presented at the international seminar on misconceptions in science and mathematics. Nueva York: Cornell University, Ithaca.

WANDERSEE, J. (1985). Can the history of science help science educators anticipate students' misconceptions? Journal of Research in Science Teaching, 27(7), pp. 581-597. 


\section{ANEXO I}

Haz corresponder a cada ser vivo de la cadena las características que posea de entre éstas:

- productor

- consumidor

- carnívoro

- herbívoro

- descomponedor
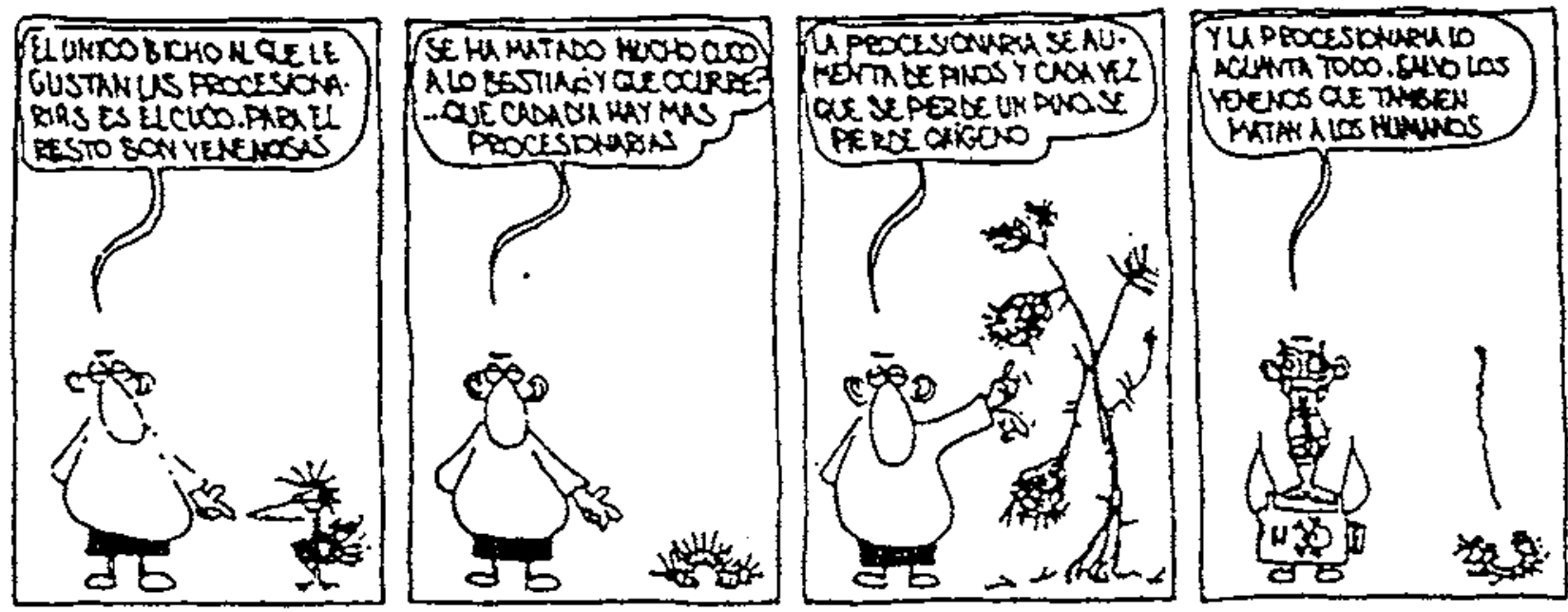

ANEXO II

En la siguiente secuencia determina el efecto que se producirá en D si decrece la población B hasta casi desaparecer. Explica tu respuesta.

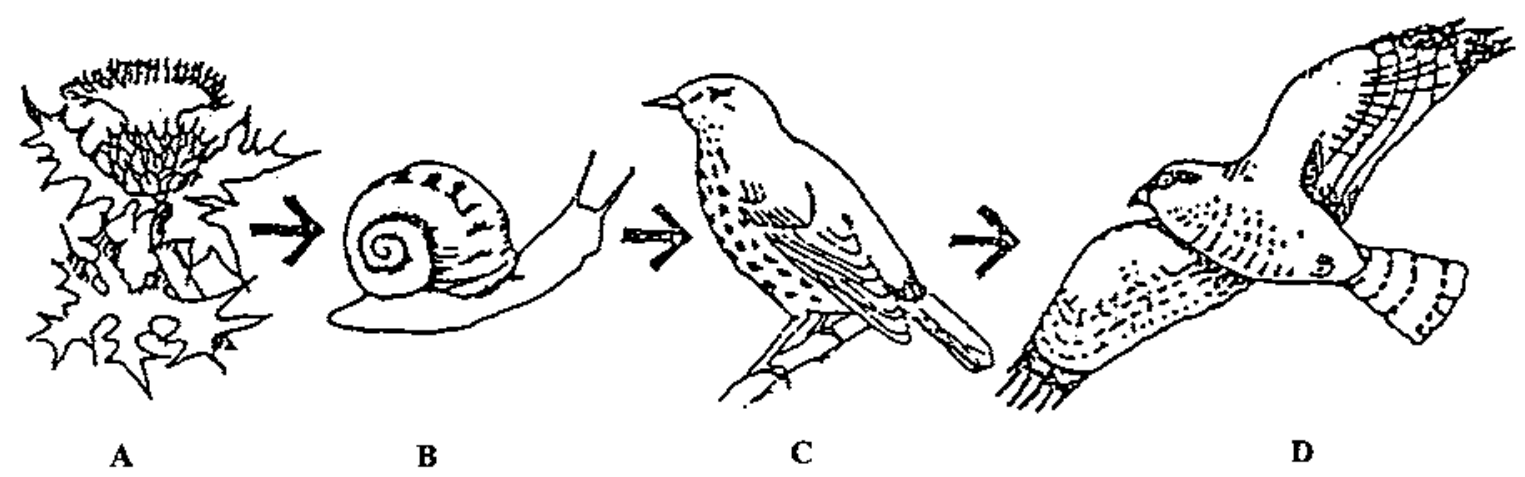




\section{ANEXO III}

Red alimentaria de una laguna en la que se presentan los caminos entrecruzados de diversos consumidores. Modificada de Alexander (1982).

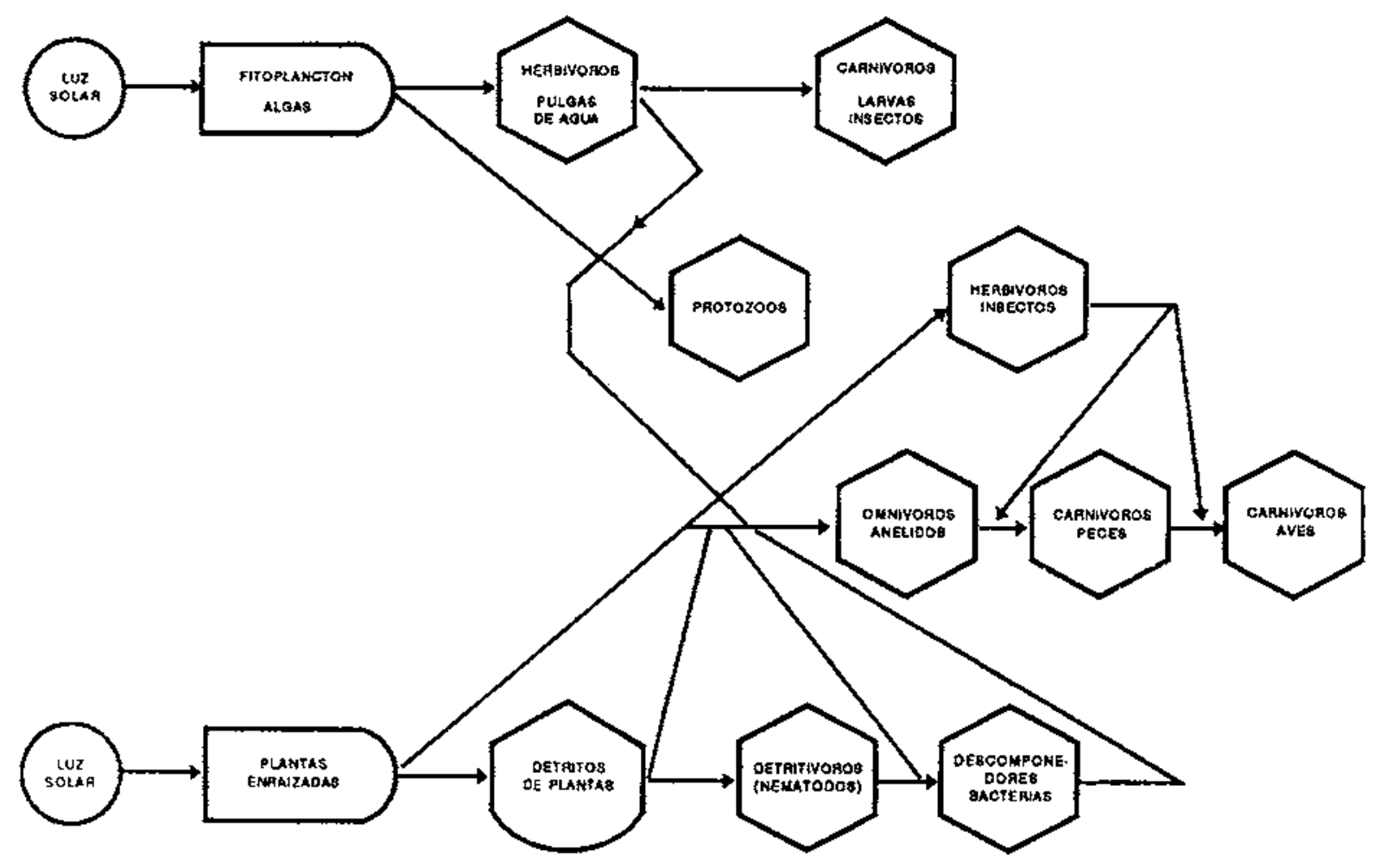

\section{ANEXO IV}

Observa la red alimentaria del esquema.

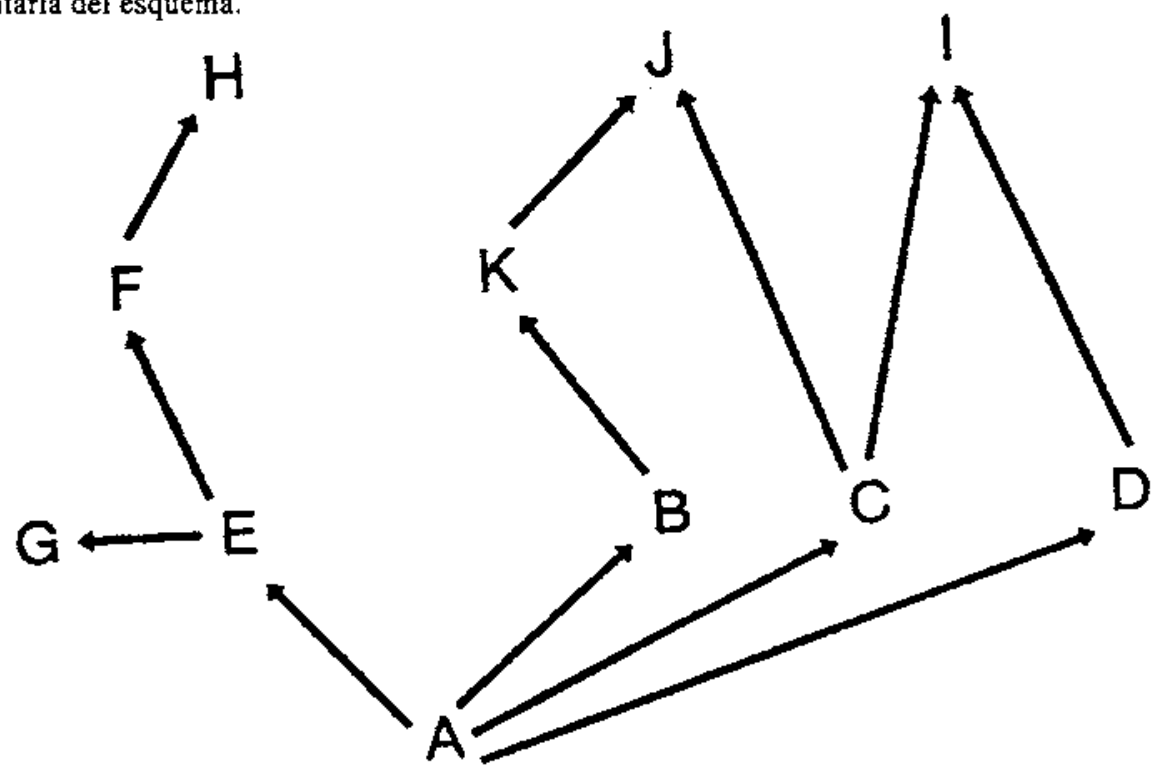

¿Qué efecto tendrá sobre la población $J$ un crecimiento súbito de la población A? Explica tu respuesta. ¿Qué efecto tendrá en la población $\mathrm{K}$ un rápido crecimiento đe la población I? Explica tu respuesta. 
